\title{
LE POIDS DES MOTS : EN ÉCOUTANT ET EN LISANT ALICJA KACPRZAK
}

\author{
Pierre MARILLAUD \\ Université Jean Jaurès de Toulouse
}

\begin{abstract}
Since Alicja Kacprzak came to four sessions of the Conference in Albi, we wanted to take part in the homage paid to her by the University of Lodz on her $6^{\text {th }}$ birthday. In 2008 she studied political propaganda posters. In 2012 she analyzed the phenomenon of insult. In 2013 she addressed the topic of medical discourse as authoritative speech. In 2014 Alicja Kacprzak and Andrzej Napieralski broached the subject of the culture and values of the world of football. These four presentations are the main theme of our article.
\end{abstract}

Merci à Alicja Kacprzak dont les mots qu'elle nous fit entendre chaque fois qu'elle vint à Albi, pesaient tout leur poids d'intelligence, de culture et d'humanité.

Dans la communication qu'elle donna en 2012 au Colloque international d'Albi Langages et Signification dont le thème était " La mauvaise parole », Alicja Kacprzak aborda la problématique de l'insulte, la place finalement assez importante qu'elle a occupée au cours de l'histoire et qu'elle occupe toujours dans la communication humaine ${ }^{1}$. Insultare en latin veut dire en premier sens 'sauter sur, ou dans, ou contre', voire 'frapper des pieds'. Mais il signifie également 'se démener avec insolence, être insolent, braver ( + datif), donner cours à son insolence, insulter', nous dit le dictionnaire latin-français Gaffiot ${ }^{2}$. Jusqu'au

\footnotetext{
${ }^{1}$ Voir Kacprzak (2013 : 43-50).

${ }^{2}$ Voir Gaffiot (1934 : 835).
} 
XIII ${ }^{\mathrm{e}}$ siècle insulter signifiait 'faire assaut'. L'étymologie n'explique pas tout, mais il est clair que l'agressivité et la violence entrent dans le sémème du lexème insulte. En termes sémiotiques on remarque que le sujet injuriant est un sujet de l'être, un sujet d'état conjoint à la violence (être insolent) et en même temps un sujet du faire (insulter, braver). C'est-à-dire que dans l'insulte il y a une sorte de défi jeté à l'autre, de tentative de le blesser, le ridiculiser, mais en donnant cours à son insolence, l'insulteur se met en position difficile par rapport aux règles de comportement dans la société. C'est-à-dire qu'au niveau des relations intersubjectives le « vous en êtes un autre » ne se justifie jamais, car l'insulte correspond toujours à une rupture dans le discours argumenté et déprécie toujours son auteur. Dans l'univers des valeurs l'insulte est toujours dépréciée et c'est la raison pour laquelle dans l'aristocratie la réponse à l'insulte ne peut se régler que par le duel. C'est le fonctionnement du code de l'honneur. Il n'est pas pensable de répondre à une mauvaise parole par une mauvaise parole. Comme le montre très bien Alicja Kacprzak dans l'article précité, la situation de communication joue un rôle très important dans la profération de l'insulte puisqu'un terme valorisant à un moment donné en un lieu donné peut devenir une insulte en d'autres circonstances.

Nous pouvons en donner un exemple en considérant l'attitude d'Alceste dans Le Misanthrope de Molière. Dans la scène 1 de l'acte I de la pièce, Alceste affirme à plusieurs reprises qu'il est un homme d'honneur et n'hésite pas à le dire à son ami Philinte, à qui il reproche de s'être montré aimable avec un personnage de la Cour à ses yeux peu intéressant :

Alceste :

[qu'il devrait] mourir de honte ;

Une telle action ne saurait s'excuser,

Et tout homme d'honneur, s'en doit scandaliser.

(Acte I, scène 1, vers 14 à 16)

Philinte répondra un peu plus loin dans le texte :

Et parfois, n'en déplaise à votre austère honneur, Il est bon de cacher ce qu'on a dans le cœur.

Serait-il à propos et de la bienséance

De dire à mille gens tout ce que d'eux on pense ?

Et quand on a quelqu'un qu'on hait ou qui déplaît,

Lui doit-on déclarer la chose comme elle est?

(Ibid., vers 75 à 80 ) 
Certes il n'y aura pas insulte entre Philinte et Alceste ; ce sont deux aristocrates qui savent se conduire, mais ces quelques vers expliquent pourquoi Alceste va répondre à Philinte, qui lui explique que par son comportement de franchise absolue, il risque un procès : « J'aurai le plaisir de perdre mon procès » (ibid., vers 196).

Quand, dans la scène 2 du même acte, Oronte, amant de Célimène, mais qui n'est pas payé en retour car elle est amante d'Alceste, vient lire un sonnet destiné à une femme qu'il aime, Alceste démolit complètement le sonnet en en montrant le côté ridicule. Oronte lui fera un procès et Alceste sera convoqué par la justice. «J'ai pour moi la justice et je perds mon procès ! » (acte $V$, scène 1, vers 1492). Il perdra également l'amour de Célimène qui ne peut plus le supporter :

\section{Célimène :}

Moi, renoncer au monde avant que de vieillir,

Et dans votre désert aller m'ensevelir !

(Acte V, scène 4, vers 1769-1770)

Si nous avons tenu à évoquer la pièce de Molière Le Misanthrope, c'est parce que nous pensons que si Alceste est un atrabilaire de tempérament coléreux, irritable et jaloux, cela n'explique pas pourquoi il perd ses procès volontairement, ou presque. C'est une question de code social qui est en jeu, voire de l'artificialité des codes sociaux de la Cour par opposition au code, désormais démodé, de l'aristocratie qui a son origine dans le code de la chevalerie médiévale. En effet un aristocrate ne peut relever un défi ou laver son honneur que par l'épée qu'il est seul à avoir le privilège de porter à son côté, et non par un procès. Or le roi en regroupant la noblesse autour de lui à Versailles, en la mettant au «parking », la transforme en une société mondaine, une société de cour, où les intrigues amoureuses et politiques, le jeu, la conversation, sont devenues des activités essentielles. Alceste est complètement décalé dans ce monde de la préciosité.

Cette épée, symbole de l'aristocratie, le bourgeois M. Jourdain la voudra, et pour cela il fera venir le maître d'armes qui n'hésitera pas à lui dire :

Et c'est en quoi l'on voit de quelle considération nous autres nous devons être dans un État, et combien la science des armes l'emporte hautement sur toutes les autres sciences inutiles, comme la danse, la musique [...].

\section{(Le Bourgeois gentilhomme, acte II, scène 3)}

Le maître d'armes est interrompu par le maître à danser qui tient à ce que l'on parle de la danse avec respect. Lorsque que l'on met les deux pièces en regard l'une de l'autre, on constate que Jourdain s'accapare de tous les 
signifiants de l'aristocratie : l'épée, la danse (telle qu'on la pratique à la Cour), la musique, la philosophie, le pouvoir en invitant le Grand Turc qui le fera " grand mamamouchi », bref tout ce dont il se sent frustré. N'oublions pas que plus tard le bourgeois Voltaire n'acceptera jamais d'avoir été bâtonné. Dans cette société de la monarchie absolue, on ne lave son honneur qu'entre aristocrates, et il n'est pas question pour un noble d'épée de croiser le fer avec un bourgeois ou un homme du peuple. Nous ne nous étendrons pas plus longuement sur Le Misanthrope ni sur Le Bourgeois gentilhomme, mais le tableau peint par Molière de la société de son temps dans ces deux pièces peut nous conduire à constater qu'en 1789 Jourdain l'emportera sur Alceste, et ce sans attribuer à Molière le moindre sens prémonitoire de l'Histoire...

La communication de l'insulte dans la société du XVII siècle en France restait encore très marquée par les valeurs et les codes de la société féodale, même si Richelieu, dans la première moitié du siècle, avait interdit le duel pour des raisons politiques. Un procès ne pouvait pourtant laver l'outrage d'un sujet du roi de naissance et de haut rang, et il faudra attendre la Révolution française pour qu'une même justice et une même loi concernent, du moins théoriquement, non plus les sujets mais les citoyens. Alicja Kacprzak, dans la communication qu'elle donna au XXXIII colloque d'Albi (Kacprzak, $2013: 47$ ), cite le scandale provoqué en 2008 par la banderole déployée par les supporters de l'équipe du Paris Saint-Germain qui jouait contre celle de Lens : «Pédophiles, chômeurs, consanguins : bienvenue chez les Ch'tis ». Elle montre "l'importance du cadre social et historique dans lequel se réalise l'acte d'insulter » (ibid.), et c'est à partir de ce même point de vue que nous avons très schématiquement comparé les deux pièces de Molière.

De la banderole, nous pouvons remonter à l'affiche car en 2008, Alicja Kacprzak avait participé au XXIX colloque d'Albi ayant pour thème « L'humour, l'ironie et les discours ». Elle y traita « Affiche de propagande ou comment ridiculiser l'ennemi politique » dont le texte figure dans les actes du colloque publiés en $2009^{3}$. Certes le terme " propagande » n'a pas toujours signifié un discours connotant sinon le mensonge, du moins l'exagération, l'enjolivement de la réalité, à des fins politiques, car, comme son étymologie le suggère, il s'agissait d'abord de la congregatio de propaganda fide, c'est-à-dire de « l'institution pour la propagation de la foi chrétienne ». Les politiques comme les religieux furent souvent des experts en propagande. Le texte que nous allons citer n'est pas tiré du discours de la propagation de la foi, mais du Manuel de l'ouvrier chrétien publié en 1850 avec l'approbation de Marie-Dominique-Auguste Sibour, archevêque de Paris :

\footnotetext{
${ }^{3}$ Voir Kacprzak (2009 : 357-367).
} 
Il nous reste, ouvriers chrétiens, à vous mettre en garde contre un dernier ennemi, la politique, oui, la politique qui dégrade l'ouvrier en le jetant dans un ordre de choses dont il n'a pas étudié les principes ; elle ne lui donne pas les connaissances indispensables dans cette position nouvelle, et lui ôte les moyens d'exercer avec fruit et honneur son ancien état. Un travailleur qui a pris le goût des clubs, des journaux et de la polémique gouvernementale, prend en aversion sa condition ; son avenir est perdu ; l'esprit de coalition et d'émeute s'empare de lui, il ne peut plus se plier aux règles de l'atelier. La plupart de ceux qui figurent comme acteurs au milieu des factieux contractent des habitudes d'oisiveté, deviennent arrogants, intraitables, turbulents ; et plus tard se montrent mauvais maris et mauvais pères. (Gaume, $1850: 39$ )

Le texte va jusqu'à culpabiliser les ouvriers qui en se révoltant, en cessant le travail provoquent « la mort du commerce et de l'industrie », le grand capitaliste ne voulant pas :

Aventurer ses fonds pour commencer de vastes entreprises au milieu d'une société où l'insurrection est, pour ainsi dire, en permanence. Le consommateur, inquiet pour l'avenir, écoute la voix de la prudence ; il réduit toutes ses dépenses, il abandonne en entier l'achat des objets de luxe, il se loge plus à l'étroit : il s'habille, ils se meuble avec plus de modestie. (Gaume, ibid.)

Bel exemple d'une propagande qui va jusqu'à la culpabilisation des ouvriers non soumis ! Il est clair que le souvenir encore très proche de la révolution de 1848 hante la prose de l'auteur de ce manuel. À la différence des affiches et textes commentés par Alicja Kacprzak, le texte que nous venons de citer ne cherche pas à ridiculiser ou caricaturer, mais il a en commun avec eux le sentiment de supériorité exprimé par l'auteur à l'endroit d'ouvriers qui n'ont pas à se mêler de politique, donc de ce qui les concerne pourtant autant qu'un bourgeois ou un noble... Si nous commentions ici le texte complet du manuel que nous avons cité, c'est la peur, la menace de l'enfer, qui prennent la place de la raillerie pour propager ce qui n'est qu'une doctrine parmi d'autres.

Il n'est pas question de relativiser l'importance de la propagande communiste en Pologne (et ailleurs) après la Deuxième Guerre mondiale, mais remarquons que tous les régimes totalitaires se substituent à toute pensée libre et individuelle qu'ils considèrent comme une atteinte morale aux systèmes respectifs qu'ils défendent. La France a connu cela avec le régime de Pétain, l'Italie avec Mussolini, l'Espagne avec Franco, et la liste serait encore très longue, mais l'aspect le plus effroyable de la propagande fut atteint par le régime nazi qui fit entrer l'amour dû au Führer dans 
l'appareil judiciaire du Reich et considéra l'extermination de la race juive comme nécessaire au redressement de l'Allemagne, et même de l'humanité ! Les nazis ne furent pas les seuls à massacrer des populations, et non seulement l'URSS, mais la plupart des pays européens ont leur part, souvent beaucoup plus importante qu'on ne le pense, au massacre général de millions d'innocents, la France comprise. Mais aucune nation n'a brandi la race maudite comme devant être exterminée, allant jusqu'à multiplier les expériences médicales sur des bébés et enfants juifs avant de les jeter dans les fours crématoires. C'est-à-dire que la propagande peut conduire à l'horreur, voire à l'inhumain. Et la grande question qui se pose est alors : comment se fait-il que la plus odieuse des propagandes soit efficace?

L'humour et l'ironie sont des réponses, certes partielles, mais incontournables: il est bien connu qu'il faut toujours mettre les rieurs de son côté et c'est ce que font, souvent avec talent, les auteurs d'affiches et de pamphlets. Mais la propagande, quand elle se double d'idéologie en même temps que d'ironie, fonctionne d'une manière culpabilisante en désignant des ennemis responsables de tous les maux, décrits la plupart du temps comme des êtres vils. Finalement la propagande assène des « vérités ». À la fin du XVIII" siècle le terme idéologie désignait l'étude de la formation des idées dans l'esprit par les philosophes empiristes et sensualistes, mais le mot changea de sens avec Marx : toute société produit un système de valeurs, de représentations symboliques, qui masquent les rapports de production économique en créant une illusion à laquelle les prolétaires sont soumis. Les idéologues comme Helvétius, Condillac, Condorcet, abandonnant d'une certaine façon la philosophie des Lumières, donnèrent au terme idéologie ses lettres de noblesse, d'autant qu'ils allèrent jusqu'à appliquer les mathématiques aux sciences sociales. Ils jouèrent un rôle important sous la Révolution, mais Marx et Engels considérèrent que certaines de leurs idées trompaient le peuple en lui proposant des systèmes de valeurs qui n'étaient que l'image inversée de la réalité, d'où l'idéologie marxiste qui prétendit représenter la réalité propre à une classe sociale. Puisqu'il est question du poids des mots, le mot idéologie a fini par prendre des connotations péjoratives, signifiant de plus en plus un système nébuleux et vague d'idées. Chez les sémioticiens greimassiens l'idéologie se différencie de l'axiologie. Quand on considère l'univers des valeurs, deux formes d'organisation sont prises en compte :

1. Les valeurs se présentant sous forme de système comme des taxinomies dont les articulations paradigmatiques sont désignées comme des axiologies. L'axiologie c'est donc la description des systèmes de valeurs, qu'elles soient morales, logiques ou esthétiques, qui relèvent de la structure profonde. Toute catégorie peut être axiologisée du fait de l'investissement des deixis positive et négative par la catégorie dite thymique (euphorie $v s$ dysphorie). 
2. Les valeurs dont les systèmes sont liés par des articulations syntaxiques sont considérées comme des potentialités de procès et peuvent être désignées par le terme idéologie. Lorsqu'un sujet individuel ou collectif prend en charge des valeurs de l'univers sémantique collectif, en les modalisant par le vouloir être et le vouloir faire, on parle d'idéologie au niveau de la structure de surface.

Il est clair que les affiches qui illustrent l'article d'Alicja Kacprzak, en caricaturant " l'ennemi du peuple » sont le résultat d'une manipulation, d'un " faire faire ", du sujet opérateur qui se présente lui-même comme le garant de la valeur qui symbolise son destinataire, à savoir le peuple. Tous les systèmes totalitaires utilisent ce procédé, car il réduit à néant la possibilité de dialogue en mettant en situation d'absurdité le peuple même qui ignorerait où se situe son intérêt premier ! L'idéologie religieuse fonctionne de la même façon, même si chez Pascal, l'idée de «l'âme jetée dans le corps » débouche sur la condamnation de celle-ci à ne trouver que nombre, temps et dimensions. Pascal oppose notre connaissance de l'existence et de la nature du fini, à notre connaissance de l'infini mais liée à notre ignorance de sa nature. C'est l'exemple là de la possibilité pour notre raison de se poser successivement les enchaînements logiques, mais le constat d'ignorance de la nature d'un monde qui s'opposerait au monde fini le pousse à un ultime argument : le pari. Mais le pari n'est pas le chemin de la foi et la raison devra se soumettre. Bien sûr les systèmes totalitaires peuvent arguer d'une foi en eux, mais l'argument serait faible et, à l'opposé de la dialectique pascalienne, ce qu'ils affirment c'est le caractère objectif, scientifique de leur démarche ou, dans les cas de leurs alliances avec l'Église, leur soumission apparente à cette église qu'ils protègent et dont ils appuient les enseignements : le politique et le religieux sont alors imbriqués l'un dans l'autre et les États de ce type n'ont même plus à se justifier!

En juillet 2013, Alicja Kacprzak revint au colloque d'Albi où elle donna une très subtile communication sur le « discours médical » dont l'ethos lui paraît être un cas particulier du discours d'autorité (thème du colloque). Elle y analysa le discours de l'éminent chirurgien du Moyen Âge, Guy de Chauliac, et celui du grand médecin de la Renaissance, Ambroise Paré. Elle avance l'hypothèse :

que si l'on parle de l'autorité, c'est parce qu'elle se réalise dans toutes les interactions, et en concerne dans la même mesure tous les participants, tous les acteurs concernés étant initialement investis d'une même importance. S'il est donc vrai que le locuteur bâtit son image, il est indiscutable que le récepteur, en dernière instance, la construit dans son for intérieur. Le discours n'a donc pas d'autorité en lui-même, mais il est investi de la valeur 
que l'auditoire, le destinataire, lui reconnaît. C'est pourquoi il n'est pas possible d'évaluer dans l'absolu l'efficacité de telle ou telle argumentation. En effet, tels arguments valables pour un public averti, ne le sont pas pour un récepteur moins savant, voire inversement. (Kacprzak, 2014 : 322)

Nous partageons ce point de vue, mais il faut bien reconnaître qu'il est des discours d'autorité qui ne sont pas censés susciter une réaction, un jugement du destinataire : les textes dits sacrés, qui restent à notre point de vue personnel des outils de manipulation des masses. Mais ils ne sont pas les seuls car les manuels scolaires, même quand ils prétendent ouvrir l'esprit des élèves, ont tendance à asséner comme des vérités, les jugements de certains critiques et auteurs. Il s'agit en fait de discours d'autorité fonctionnant dans un environnement fortement hiérarchisé et orienté, comme par exemple celui de l'éducation en France.

Nous ne prendrons qu'un exemple banal : tout élève de lycée du $\mathrm{XX}^{\mathrm{e}}$ siècle qui suivait la filière classique était, nous oserons dire " contraint », d'admirer, ou d'aimer, ou de considérer Platon comme une autorité incontournable de la philosophie grecque. Certes ce philosophe reste à nos yeux (peut-être avons-nous été trop bien dressé ?) un écrivain et un philosophe important, mais son autorité était telle à l'époque que nous citons qu'il était impensable de relativiser, voire critiquer le maître d'Aristote, du moins tant qu'on n'avait pas acquis les titres nécessaires pour avoir le loisir d'émettre des avis personnels. Bien sûr nous ne sommes pas en train d'assimiler le discours de Platon à un discours de propagande, mais il fallait arriver dans l'enseignement supérieur, et avoir le privilège d'écouter certains professeurs capables de nous aider à prendre la distance avec le philosophe en question, pour accéder à une forme de pensée libre et non culpabilisée. Et pourtant que n'a-t-on dit, et à juste titre, sur Hitler ordonnant qu'on brûle des livres, alors que Platon voulait qu'on brûlât l'œuvre de Démocrite, qu'on censurât Homère et des pièces tragiques. Que Platon ait été croyant en un dieu (le Dieu) comme il l'exprime dans le Timée (30c-31b) ne nous gêne pas, mais qu'il propose une loi qui punirait tout comportement impie aussi bien en parole qu'en acte, et qu'un magistrat qui ne voudrait pas appliquer cette loi serait lui-même condamné, comme il l'exprime dans le livre $\mathrm{X}$ des Lois, voilà des points qui ne furent pas mis en relief par la critique platonicienne, et seuls les hellénistes et les initiés à un haut niveau de la pensée philosophique grecque savaient et se gardaient la plupart du temps de faire état de ces «monstruosités de la pensée ». Lucrèce nous dit que Démocrite se suicida quand il sentit que le poids de l'âge portait atteinte à sa mémoire, mais Platon dans le Phédon (62 c) estime qu'il ne faut pas se tuer avant que le Dieu en impose la nécessité... Nous ne sommes ni spécialiste, ni vraiment compétent pour juger d'une œuvre qu'il fallait cependant avoir quelque peu fréquentée si l'on voulait passer le 
cap du baccalauréat de philosophie, mais nous avons le souvenir de ce monument de la philosophie qu'était à nos yeux l'œuvre de Platon. Faut-il préciser que Platon défend l'esclavage, que dans La République les élites ne peuvent se mélanger avec les autres classes de la société, que les enfants mal formés doivent vivre dans un endroit reculé et caché de la cité, etc.

Nous constatons alors qu'un discours d'une autorité incontestée portait en lui tous les germes de la théocratie, de la dictature, or, pour reprendre l'expression d'Alicja Kacprzak, nous n'étions pas un «public averti ». Sur ce plan, il semble qu'aujourd'hui les lycéens aient la chance d'être plus tôt informés des sommets et des horreurs de l'œuvre de Platon. Rappelons que l'Église catholique a toujours favorisé la lecture de Platon car sa croyance en un seul Dieu et en l'éternité de l'âme sont des arguments très forts de la pensée chrétienne.

Dans la communication qu'Alicja Kacprzak et Andrzej Napieralski donnèrent en 2014 au XXXV Colloque international d'Albi, ils se livrent au décodage des messages des écharpes des ultras des clubs de football, et les mettent en relation avec ce phénomène de continuité qui concerne les croyances et les religions. Malgré ce qui distingue ces messages des croyances et religions, des similitudes profondes apparaissent. Reprenant la thèse développée par Thomas Luckmann dans The Invisible Religion: The Transformation of Symbols in Industrial Society (publié en anglais en 1967), les auteurs de la communication reprennent cette idée que la religion finit par être remplacée par le religieux qui finit par doter différents phénomènes laïques de fonctions quasi religieuses, l'analyse des messages des écharpes en étant l'illustration. Il est clair que le message « Tu n'auras pas d'autres clubs que moi » est un exemple frappant qui nous renvoie presque à l'Ancien Testament et à un propos injonctif tel qu'on aurait pu l'entendre sur le Sinaï ! Cette communication, intéressante à de nombreux titres, prend une résonance très particulière dans notre monde troublé par une violence se manifestant un peu partout. Après une analyse très détaillée à laquelle nous renvoyons nos lecteurs ${ }^{4}$, les auteurs nous montrent :

que les écharpes de supporters constituent un phénomène social global, une sorte de continuum socioculturel, étant donné leur fonctionnement généralisé dans plusieurs pays, aussi bien en tant qu'attribut de grands clubs connus que de clubs tout petits et insignifiants. [...] En ce sens, les écharpes des supporters fanatiques considérées parfois comme une pratique ludique anodine constituent en fait, grâce à leur contenu visuel et textuel, un important marqueur identitaire à valeur totémique. (Kacprzak, Napieralski, $2015: 155)$

\footnotetext{
${ }^{4}$ Voir Kacprzak, Napieralski (2015 : 147-157).
} 
Cette communication est un exemple de la lucidité avec laquelle Alicja Kacprzak et Andrzej Napieralski mettent en évidence un phénomène socioculturel qui, dans le monde hyperorganisé, cadré, numérisé, de la société contemporaine, crée une sorte de faille, d'où émergent, comme les fumerolles ou la lave d'un volcan, une violence et un sacré qui sont les reflets des tensions qui agitent en permanence le sous-sol de nos civilisations...

Pour conclure, nous dirons, parce que nous avons eu le privilège de connaître, d'écouter, de lire Alicja Kacprzak, que nous savons à quel point elle éveille chez tous les destinataires de son discours la sensibilité aux valeurs de l'humanisme, à la générosité, à la tolérance, au respect de l'autre et nul doute qu'elle transmet à ses étudiants ce qui nous permet de rester fier, malgré le pessimisme ambiant, d'une certaine conception de l'Humanité. Qu'il nous soit permis de la remercier d'être venue participer à nos colloques où sa compétence se manifesta avec autant d'intensité que cette gentillesse naturelle qui impressionne et qui enveloppe lorsqu'on a le privilège de lui parler.

\section{Références bibliographiques}

\section{- linguistiques}

GAFFIOT, Félix (1934), Dictionnaire illustré latin-français, Paris, Hachette.

GAUME, les frères (1850), Manuel de l'ouvrier chrétien, édité par les frères Gaume, avec approbation de Monseigneur Sibour, archevêque de Paris, Saint-Germain-en-Laye, Imprimerie de BEAU.

KACPRZAK, Alicja (2009), «Affiche de propagande ou comment ridiculiser l'ennemi politique », in Humour, ironie et les discours. Actes du XXIXe Colloque d'Albi Langages et Signification (P. Marillaud, R. Gauthier éds), Toulouse, Éditions CALS/CPST, Université de Toulouse-LeMirail, pp. 357-367.

KACPRZAK, Alicja (2013), " De la socio-pragmatique de l'insulte : point de vue interlingual », in La mauvaise parole. XXXIII C Colloque d'Albi Langages et Signification (P. Marillaud, R. Gauthier éds), Toulouse, Éditions CALS/CPST, Université de Toulouse-Le-Mirail, pp. 43-50.

KACPRZAK, Alicja (2014), « De l'autorité construite dans le discours médical », in Discours d'autorité et discours de l'autorité. Actes du XXXIV Colloque international d'Albi Langages et Signification (P. Marillaud, R. Gauthier éds), Toulouse, Éditons CALS/Université ToulouseJean-Jaurès, pp. 315-325.

KACPRZAK, Alicja, NAPIERALSKI, Andrzej (2015), « Culture et valeurs du monde du football : les messages des écharpes des ultras », in Cultures et valeurs. Actes du XXXVe Colloque d'Albi 
(P. Marillaud, R. Gauthier éds), Toulouse, Éditons CALS/Université Toulouse-Jean-Jaurès, pp. 147-157.

LUCKMANN, Thomas (1967), The Invisible Religion: The Transformation of Symbols in Industrial Society, New York, Macmillan.

\section{- littéraires}

MOLIÈRE (2011 [1666]), Le Misanthrope, Paris, Gallimard.

MOLIÈRE (2003 [1670]), Le Bourgeois gentilhomme, Paris, Larousse.

PLATON (1940-1943), Euvres complètes, vol. 1-2, trad. fr. L. Robin, Paris, Gallimard. 